\title{
RF Control of Biological Systems: Applications to Wireless Sensor Networks
}

\author{
Hooman Javaheri ${ }^{1}$, Guevara Noubir ${ }^{1}$, and Sanaa Noubir ${ }^{2}$ \\ 1 College of Computer and Information Science, \\ Northeastern University, Boston MA 02115, USA \\ \{hooman, noubir\}@ccs.neu.edu \\ 2 Division of Infectious Diseases, Faculties of Medicine and Science, \\ University of British Columbia, Vancouver BC V5Z 3J5, Canada \\ sanaa@interchange.ubc.ca
}

\begin{abstract}
We present a vision and preliminary results for a combined RF-Biological Systems where the Electro-Magnetic energy of RF signals is transduced into control-signals for biological signaling-processes. We show how such systems can be used to build extremely low-power sensor networks. We present our approach and propose a system architecture constituted of a passive RF Front End (RF-FE), Bio-Mechanical Signal Interpreter (BMSI), and a Low Power Sensor Node. We show preliminary results for the performance of our RF-FE prototype, outline design alternatives for the BMSI, and summarize theoretical results for characterizing the BMSI components performance.
\end{abstract}

Key words: RF, biological system, wireless sensors, energy harvesting

\section{Introduction}

One of the major challenges in conserving energy in wireless sensor networks is to reduce the energy consumption of the RF receiver. Today's techniques rely on periodically waking up the receiver to synchronize and respond to the requests of a master node. Ideally, a sensor node should go in a full-sleep mode that consumes zero energy and only wake up on external requests or events. We contend that the energy efficiency of biological systems can be harnessed to design and build a combined RF-biological nano-power sensing device, bringing us closer to the ideal wireless sensor node. This device can go in a full sleep mode but can still be waken up using a fairly long-range RF signal. Our idea is to transduce a weak Electro-Magnetic (EM) signal into a biological signal (e.g., change in concentration of ions in the neighborhood of excitable cells) and use a biological device to demodulate the information embedded in the original EM signal. This task is carried out by the Bio-Mechanical Signal Interpreter (BMSI). In the context of wireless sensor nodes, the main functionality of the BMSI would be to recognize the sensor node address and generate a signal to wake up the main sensor node. 
Related Work A significant amount of research has been done to investigate the problem of energy conservation in wireless sensor networks. Previous research focused on the design of low power hardware and development of efficient algorithms for low duty cycle and coordinated wake up protocols [1, 2, 3]. Recently, several energy harvesting techniques were proposed to extend the lifetime of wireless nodes $[5,6,7]$. Over the last few years, biochemically-engineered molecular communication has gained significant interest [4]. The effects of the very low frequency electro-magnetic signals on cell metabolism were explored [8]. However, most previous work focussed on the thermal effects of high frequency RF waves on biological systems[9]. To the best of our knowledge there is no previous work to use RF signaling to modulate a biological signaling process that can ultimately trigger the wake up of a sensor node.

In Section 2, we outline our approach and the proposed system architecture along with components. In Section 3 we show some preliminary results of our prototype for the passive RF Front-End. In Section 4, we provide more details about the BMSI component, the blueprint for its node address recognition functionality, and alternative designs. We summarize the theoretical characterization for the achievable change in ion concentration that is required to trigger an amplifiable inter-cellular signaling mechanism (e.g., calcium ions) and discuss some of the challenges. We conclude with a discussion of related work and directions for future work.

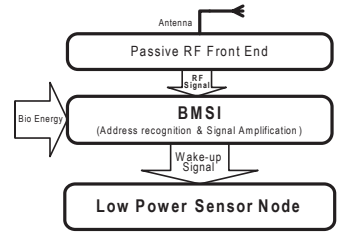

(a)
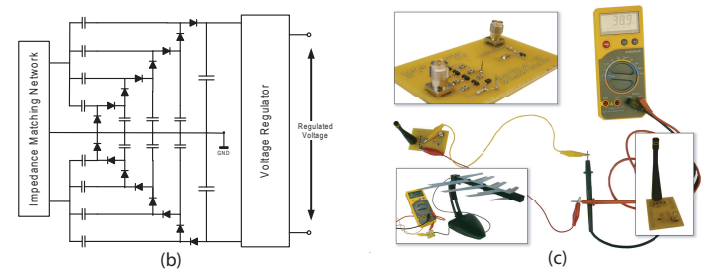

Fig. 1. (a) System components. (b) The RF energy harvester unit schematics. (c) The implemented device.

\section{Approach and System Architecture}

In an ideal wireless sensor network, the sensor nodes remain in a full sleep mode while being responsive to remote queries sent over the RF signal. This can be achieved using an extremely low power wake-up system to trigger a Low Power Sensor Node (LPSN). The received RF signal from the antenna is extremely weak, thus cannot be used directly to wake up the LPSN. We propose to combine the RF energy harvesting mechanism with the energy efficiency of biological processes. The system accumulate the energy of the RF signal and transduces it 
into a biological signal. The incoming signal information is processed using the energy-efficient biological system and if necessary triggers the wake up of the LPSN. We break down the design of the proposed system into three components (See Figure 1.a): (1) a passive RF Front End (RF-FE) to harvest and detect the weak remote RF signal, (2) a Bio-Mechanical Signal Interpreter (BMSI) to efficiently execute the signal demodulation and generate the wake-up signal, and (3) a Low Power Sensor Node (LPSN).

\section{RF Front End}

In order to add the desired features, we modify the design of the wireless node's RF Front End. We use an array of RF energy harvesting circuits to scavenge energy of the incoming signal RF signal. An RF Energy harvester consists of a sharp filter connected to a multistage voltage multiplier followed by a voltage regulator (See Figure 1.b). The RF energy harvester accumulates the energy of the RF signal in the frequency band of its filter. In our model, nodes are communicating wirelessly using Multiple Frequency Shift Keying (MFSK) modulation with alphabet size of $M$. The RF-FE includes $M$ energy harvester circuits each of which is tuned to one of the MFSK frequencies. Hence, the output of the RF-FE will be a set of $M$ regulated DC voltages, $V_{i}=V_{\text {reg }}$ if the $i$ th frequency component of the RF signal is present, otherwise 0 . We built and tested an RF energy harvester unit using a 4-stage modified Greinacher voltage multiplier with the schottky diodes. Figure 1.c depicts the preliminary performance evaluation result of the harvester unit.

\section{Bio-Mechanical Signal Interpreter}

As illustrated in Section 3, the RF-FE performs a partial demodulation on the RF signal. However, the output of the RF-FE still needs to be decoded and amplified in order to trigger the LPSN appropriately. Since the only source of electrical energy in our design is the nano-power RF-FE output, it is not feasible to use conventional active components, which consume considerably higher amounts of energy. In other words, the BMSI is basically an electrically passive decoder-amplifier. The BMSI converts the weak electrical signals of the RF-FE into Bio-Mechanical signals (e.g., change of the concentration of a specific ion as a biological stimulator). The signal amplification and decoding processes are performed in the biological environment, thus very energy efficient. Finally, the wake-up signal is generated and sent to LPSN. In the following, we break down the design and functionality of the BMSI into Address Recognition mechanism and Biological amplification.

\subsection{Address Recognition Maze}

The key functionality of the BMSI will be an address recognition mechanism. In our wireless sensor network model, we assume that the received signal is a fixed 
request (e.g., wake-up signal) sent from a single transmitter to a specific sensor - we don't want to wake up all the nodes within the range of the transmitter. Therefore, only the ID of the sensor is coded in the received signal. To implement this address recognition, we design a system based on electrically mediated ion transport in a nano-mechanical maze. We connect the RF-FE outputs to the embedded electrodes in order to generate controlled electrical fields. These fields guide the motion of the ions through the maze. We encode the node ID in the nano-maze structure such that the concentration of the ions will significantly increase if and only if the output signals from the RF-FE matches the node ID. (See Figure 2). Each RF-FE output corresponds to an electrode. The maze is filled with a homogeneous aqueous solution of a target ion with initial concentration of $c_{0}$. The $F_{0}$ provides a stable horizontal electrical field that moves the ions forward in the maze. The vertical electrical fields generated by $F_{1}, . ., F_{M-1}$ determine the path on which the ions are traveling. There are two types of junctions in the maze. At 1-junction, ions will stay on the main path if a vertical field is present, otherwise they are guided to the path that leads to a dead-end. Following the same physics, at 0-junction, the ions will stay on the main path if there is no vertical field and go to the dead-end if the vertical field exists. We can simply represent the node's ID with the sequence of its maze junction types. In case the RF-FE signal matches the node ID, the concentration of the target ion at the end of the maze keep increasing until it reaches the Nernst equilibrium [10].

In order to preserve the functionality, the maze should be reset to the initial condition after each round of operation. In the absence of forwarding electrical field (i.e., $F_{0}$ ), the diffusion moves the transported ions back to form a homogeneous solution once again. However, this process might be slow in some cases (e.g. a matching wake-up signal immediately sent after a non-matching wake-up signal that pushed the ions into the dead-ends). In our solution, the transmitter sends a reset signal before sending the actual wake-up signal. The reset signal is sent on a specific frequency of the RF-FE and generates a horizontal electrical field in the opposite direction of $F_{0}$. This backward ion transport moves back the ions that might be stuck in the dead-ends and makes the system ready to operate on the actual wake-up signal.

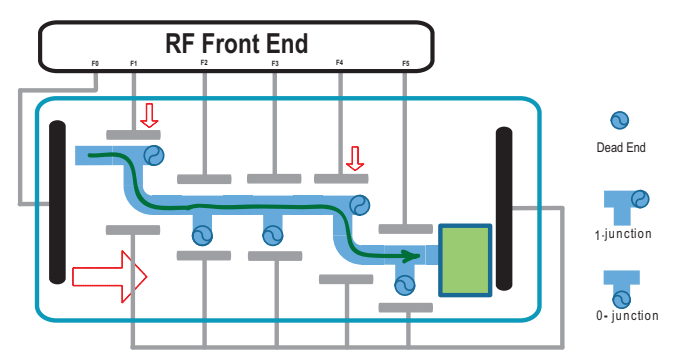

Fig. 2. The structure of the nano-maze. Arrows show the necessary electrical fields to match the node ID of 10010 . 


\subsection{Biological Component}

At the end of the maze, we place a group of excitable cells sensitive to the increase of the target ion concentration. Once it passes the threshold value of $C_{\text {thresh }}$, a desired biological control signal is created. This triggers a pre-designed chain of biochemical reactions (i.e., signaling pathway) in the biological component. The response is a chemical, mechanical or an electrical signal strong enough to trigger the LPSN directly. The energy required for the amplification is provided by the biological elements of the system (i.e., cells) which are known to be extremely power efficient [11]. The specialized cells used to perform the desired biochemical chain reaction can be genetically engineered using known synthetic biology methods [12] (e.g., BioBricks [13]).

A major challenge is how to keep the biological component alive and operational since its performance is very sensitive to the environmental characteristics. Several methods has been developed and many are under investigation to alleviate this problem. Cultured media buffered naturally by dissolved solutes in the media can allow some $\mathrm{pH}, \mathrm{CO}_{2}$ /Oxygen variation during the culture of these cell lines. The room temperature is usually ideal for slow growth [15]. In addition, cells can be grown in the presence of a low fetal calf serum concentration to allow slow growth over a long period in order to avoid reaching a high cell density that may affect cells viability. Most cell lines available from the Global Bioresource Center "ATCC" are capable to grow for up to 12 weeks provided that they have optimal growth conditions (e.g., nutrients from fetal calf serum, $p H$, temperature, $\mathrm{CO}_{2}$ /Oxygen) [15]. However, the sensitivity of animal and human cells to changes experienced in their culture media conditions is evidenced by the activation of a gene-encoded process called apoptosis or programmed cell death. Extensive research efforts have been devoted to the development of apoptosis protective mechanisms and encouraging results were obtained [16]. Experiments using over-expression of cloned anti-apoptosis genes interfering at the mitochondrial, initiator protein caspases and telomeric shortening levels have proven effective in diminishing programmed cell death in a variety of cell lines, thus increasing greatly the viability of these cells $[16,17]$. These apoptosis-resistant cells have shown to be more robust due to their enhanced ability to grow in stressfull conditions (e.g., very low nutrient media, heat shock, hypoxia) $[16,17,18]$. Immortalized cell lines are an example of candidates capable of providing the desired viability to the system. Alternatively, we

plan to explore the use of extremophile organisms, which are resilient to extreme environmental conditions [19].

Calcium Ion Energy Budget Analysis Several biological systems (e.g., human body) use calcium ions $\left(\mathrm{Ca}^{2+}\right)$ to stimulate a variety of excitable cells such as neurons and muscle cells [14]. The intercellular calcium signaling is a very good candidate for the BMSI biological mechanism. In this section, we provide a simple calculation to show that the proposed BMSI design is physically implementable. Assume we use a calcium salt solution in the BMSI and the 
target ion is $\mathrm{Ca}^{2+}$. Let the initial concentration of $\mathrm{Ca}^{2+}$ be $c^{*}$, then we have $c_{\text {thresh }}=10 \times c^{*}$. We assume the received $\mathrm{RF}$ signal matches the node ID, hence we can safely approximate the maze with a simple tube with a effective length of $l$, slightly larger than actual length of the maze. Assuming the ion concentration only changes along the length of the tube, we solved the Nernst-Planck equation for the ion flux in the steady state. Let $E, j, q, D, K_{B}$, and $T$ denote the electrical field, ion flux, charge of the ion, diffusion coefficient, Boltzmann constant, and the temperature respectively. We have:

$$
j=D\left(\frac{d c}{d x}+\frac{q}{K_{B} T}\right) E=0 \Rightarrow c(x)=c_{\text {start }} \times e^{\frac{q E}{K_{B} T}}
$$

where,

$$
c_{\text {start }}=\frac{q l E c^{*}}{K_{B} T\left(e^{\frac{q l E}{K_{B} T}}-1\right)}
$$

Here, $q=+2 e$ and $\frac{K_{B} T}{e}=\frac{1}{40} V$. Therefore, the concentration of the calcium ions at the end of the maze will be:

$c(l)=\frac{q l E c^{*}}{K_{B} T\left(e^{\frac{q l E}{K_{B} T}}-1\right)} e^{\frac{q l E}{K_{B} T}}=\frac{2 e V_{r e g} c^{*}}{K_{B} T\left(e^{\frac{2 e V_{r e g}}{K_{B} T}}-1\right)} e^{\frac{2 e V_{r e g}}{K_{B} T}}=\frac{80 V_{r e g} c^{*}}{\left(e^{80 V_{r e g}}-1\right)} e^{80 V_{r e g}}$

To reach $c_{\text {thresh }}=10 c^{*}$, we need to have $V_{\text {reg }} \approx 120 \mathrm{mV}$ which is achievable with the proposed RF-FE design.

\section{Conclusion and the future work}

We present a vision and an approach on how to utilize the power-efficiency of biological systems to build a new breed of wireless sensor networks that consumes significantly lower energy than today's wireless sensor nodes. We present the design, preliminary analysis and evaluation of a combined RF-Biological system. There are several challenges to optimize the performance of the system. The proposed bio-enabled wake up mechanism is a slow process that might take tens of seconds. This is a strong constraint on the capacity and delay of the wake up channel. It is therefore important to understand the tradeoffs between capacity, delay, and energy, in the specific context of networks of bio-enabled devices with multiple master nodes contending for the same RF channel. A solution to be considered consists of using a hybrid approach where full sleep mode and periodic wake ups are combined to satisfy the network capacity requirements, application delay, and system energy constraints. The extension of the communication model to multiple transmitter scenario that includes the mechanism for carrier sensing and collision detection is part of our future work. Accurate characterization, modeling and experiments are necessary to find the reliable biological signaling pathways used in the biological component. In addition, Extensive work needs to be done to boost the viability of biological component, thus the reliability of the system. We believe, the proposed approach may open a new way in the wireless real-time control of the biological systems with possible medical and biological applications (e.g., wireless medical treatment and drug delivery). 


\section{References}

1. Van Dam T., Langendoen K.: An adaptive energy-efficient MAC protocol for wireless sensor networks: SenSys '03. ACM, New York, NY, 171-180, 2003.

2. Polastre J., Hill J., Culler D.: Versatile low power media access for wireless sensor networks: In Proceedings of the 2nd international Conference on Embedded Networked Sensor Systems, Baltimore, MD, USA, Nov 2004.

3. Slijepcevic S., Potkonjak M.,: Power efficient organization of wireless sensor networks: ICC 2001, IEEE International Conference on Communications, vol.2, no., pp.472-476 vol.2, 2001.

4. Nakano T., Suda T., Moore M., Egashira R., Enomoto A., Arima K.: Molecular Communication for Nanomachines Using Intercellular Calcium Signaling: IEEE NANO 2005, Japan, June 2005.

5. Paradiso J.A., Starner T.:Energy scavenging for mobile and wireless electronics,: Pervasive Computing, IEEE, vol.4, no.1, pp. 18-27, Jan.-March 2005.

6. Rahimi M., Shah H., Sukhatme G.S., Heideman J., Estrin, D.: Studying the feasibility of energy harvesting in a mobile sensor network,: Proceedings. ICRA '03. IEEE International Conference on Robotics and Automation, vol.1, no., pp. 19-24 vol.1, 14-19 Sept. 2003

7. Roundy S., Wright P.K., Rabaey J.:A study of low level vibrations as a power source for wireless sensor nodes: Computer Communications, Volume 26, Issue 11, Ubiquitous Computing, Pages 1131-1144, July 2003.

8. Rosenspire A.J., Kindzelskii A.L., Simon B.J., Petty H.R.:Real-Time Control of Neutrophil Metabolism by Very Weak Ultra-Low Frequency Pulsed Magnetic Fields: Biophys J.; vol. 88(5), 33343347, May 2005.

9. Gordon, C.J.,: Normalizing the thermal effects of radiofrequency radiation: Body mass versus total body surface area: Bioelectromagnetics J., vol 8(2), 111-118, Oct. 2005.

10. Nelson P.: Biological Physics: Energy, Information and Life: W. H. Freeman \& Company, New York, NY, p. 136-145, 2004.

11. Freeman S.: Biological Science: Second Edition, Prentice Hall, NJ, 2005.

12. Lauffenburger D.A.: Cell signaling pathways as control modules: Complexity for simplicity?: PNAS, vol. 97 no. 10 5031-5033, May 2000.

13. BioBricks Foundation, http://bbf.openwetware.org.

14. Berridge M.:The AM and FM of calcium signaling: Nature, 386:759-780, 1997.

15. ATCC the Global Bioresource Center http://www.lgcstandards-atcc.org/

16. Vives J, Juanola S, Cair JJ, Gdia F.: Metabolic engineering of apoptosis in cultured animal cells: implications for the biotechnology industry: Metab Eng, 5(2):124-32. Review Apr. 2003.

17. Reddel RR. Ann: Genes involved in the control of cellular proliferative potential: NY Acad Sci, 20;854:8-19, Nov 1998.

18. Zha Y, Chen XQ, Du JZ:Cellular adaptation to hypoxia and p53 transcription regulation: J Zhejiang Univ Sci B. 10(5):404-10, May 2009.

19. Zahradka K., Slade D.,Bailone A., Sommer S., Averbeck D., Petranovic M., Lindner A.B., Radman M.: Reassembly of Shattered Chromosomesin Deinococcusradiodurans: Nature 443 October 2006, 569-573. 